\title{
Fine-scale structure of the neutral ISM in M81
}

\author{
Ioannis Bagetakos ${ }^{1}$, Elias Brinks ${ }^{1}$, Fabian Walter ${ }^{2}$ and Erwin de Blok ${ }^{3}$ \\ ${ }^{1}$ Centre for Astrophysics Research, University of Hertfordshire, UK \\ ${ }^{2}$ Max-Planck-Institut für Astronomie, Heidelberg, Germany \\ ${ }^{3}$ Mount Stromlo Observatory, Australian National University, Canberra, Australia
}

\begin{abstract}
We present an analysis of the fine-scale structure of the neutral ISM as traced via the 21-cm line of atomic hydrogen (HI) in the nearby galaxy M 81. The data show a stunning amount of detail in the form of 330 expanding shells and holes in the neutral ISM of M 81. A comparison with similar structures found in two other spirals and two dwarf galaxies (M 31, M 33, IC 2574 and Holmberg II) reveals that the ISM in M 81 shares a lot of similarities with the two spirals, whereas the structure of its ISM is different to that in dwarf galaxies. The sizes of the $\mathrm{HI}$ holes in M 81 range from $80 \mathrm{pc}$ (close to the resolution limit) to $600 \mathrm{pc}$; the expansion velocities can reach $20 \mathrm{~km} \mathrm{~s}^{-1}$; estimated ages are 2.5 to 35 Myrs and the energies involved range from $10^{50}$ to $3.5 \times 10^{52}$ ergs. The amount of neutral gas involved is of order $10^{4}$ to $10^{6}$ solar masses.
\end{abstract}

Keywords. galaxies: individual (M 81) — galaxies: ISM — ISM: structure — radio lines: ISM

M 81 forms part of "The HI Nearby Galaxy Survey" (THINGS), a survey performed with the NRAO Very Large Array resulting in data with $7^{\prime \prime}$ angular and $5 \mathrm{~km} \mathrm{~s}^{-1}$ or better velocity resolution (Walter et al. 2005). At this resolution the HI distribution shows a wealth of structure which is interpreted as being due to expanding HI shells. We conducted a search for HI shells by inspecting channel maps, analysing position-velocity cuts and looking at the integrated HI map. A total of 1212 candidate holes were detected in the HI distribution of M 81 for each of which a set of properties was determined. We selected the 330 clearest defined shells and compared their characteristics with those found in two spiral (M 31, M 33) and two dwarf galaxies (IC 2574, Holmberg II). The distributions of the size and the kinematical age of the HI shells were found to be similar for all three spirals, whereas they were clearly different from those in the dwarf galaxies, the diameter of shells in dwarf galaxies being larger than in spirals. Importantly, the amount of energy typically deposited in the ISM is the same, irrespective of galaxy type. This is ascribed to dwarf galaxies being lower mass systems which implies that for an observed velocity dispersion which is similar to that of spiral galaxies, they have a much thicker HI disk (Brinks et al. 2002). This means that a shell with the same energy input can grow larger in a dwarf galaxy than in a spiral. Also, the absence of shear in dwarf galaxies makes that structures in the ISM can persist longer.

\section{References}

Brinks, E., Walter, F., Ott, J. 2002 Bloated Dwarfs: The Thickness of the HI Disks in Irregular Galaxies (ASP-CS), 275, 57

Walter, F., Brinks, E., de Blok, W. J. G., Thornley, M. D., Kennicutt, R. C. 2005, First Results from THINGS: The HI Nearby Galaxy Survey (ASP-CS), 331, 269 